\title{
Whole-Mount Observation of Pharyngeal and Trabecular Cartilage Development in Lampreys
}

\author{
Tuoya Yao, Kaoru Ohtani and Hiroshi Wada* \\ Graduate School of Life and Environmental Sciences, University of Tsukuba, \\ Tsukuba 305-8572, Japan
}

\begin{abstract}
Because the jaw is thought to have evolved as a dorsal-ventral articulation of the anterior pharyngeal arch, knowledge of developmental patterning in the pharyngeal arch is critical to understanding the origin and evolution of the jaw in gnathostomes. It is particularly important to determine whether Agnatha already possessed developmental polarity along the dorsal-ventral axis of the pharyngeal arch. We used the Weigert staining method to examine the development of cartilage in whole-mount lamprey specimens. We found that although the transverse rods showed symmetrical patterning along the dorsal-ventral axis, the hypobranchial bar and subchordal rod showed distinct developmental patterning. Thus, our observations suggest that pharyngeal cartilage also differentiates along the dorsal-ventral axis. In addition, the parachordal rods were shifted dorsally compared to the subchordal rods. Although the development of cartilage occurred earlier in the anterior arches, the fusion of the subchordal rods occurred earlier in the posterior arches. We also noted a unique morphology of cartilage in the ninth pharyngeal arch. Our descriptions and the methods used here facilitate the observation of pharyngeal cartilage in wholemount specimens, and will aid in the study of developmental patterning in the lamprey pharyngeal arch.
\end{abstract}

Key words: lamprey, development, cartilage, jaw, pharyngeal arch

\section{INTRODUCTION}

The muscular pharynx is one of the most important vertebrate characteristics. Protochordates, such as ascidians and amphioxus, possess thin pharyngeal bars lined with cilia that produce water currents. In contrast, vertebrates have thick pharyngeal arches composed of muscle and cartilage, and water currents are produced by muscular movement of the pharyngeal arches (Mallatt, 1984). The evolution of thick pharyngeal arches is due to the emergence of neural crest cells. The enigmatic fossil Haikouella was classified as a primitive vertebrate because of the presence of thick pharyngeal arches, suggesting the presence of neural crest cells in this animal (Mallatt et al., 2003). The acquisition of a muscular pharynx allowed early vertebrates to ventilate forcefully and support a larger body size than do protochordates (Mallatt, 1996).

The extant Agnatha, i.e., lampreys and hagfishes, also possess muscular pharyngeal arches supported by cartilage. However, there are two fundamental issues concerning the early evolution of this cartilage in vertebrates and agnathans. First, although agnathan pharyngeal cartilage is derived from neural crest cells (Langille and Hall, 1988), its matrix component was said to be distinct from that of gnathostome cartilage (Wright et al., 1988); therefore, it was uncertain whether agnathan cartilage is homologous to that

\footnotetext{
* Corresponding author. Phone: +81-29-853-4671;

Fax : +81-29-853-4671;

E-mail: hwada@biol.tsukuba.ac.jp
}

of gnathostomes. The main matrix components of the lamprey cartilage are elastin-like molecules (Robson et al., 1997; Wright et al., 1988), whereas gnathostome cartilage is largely composed of fibrillar collagen. Recent reports have largely settled this issue with new evidence that lamprey chondrocytes, like those of gnathostomes, differentiate under regulation of the SoxE and SoxD genes, and that a fibrillar collagen gene is expressed in lamprey chondrocytes (McCauley and Bronner-Fraser, 2006; Zhang et al., 2006, Ohtani et al., 2008). In addition, Rychel and Swalla (2007) presented evidence that pharyngeal-bar cartilage in amphioxus and acorn worms shows similar gene expression profiles, with SoxE and collagen co-expression in the gills. These results suggest that cartilage is homologous among deuterostomes, although there are some differences in matrix components between invertebrate deuterostomes and agnathans versus gnathostomes. Differences also exist in the cellular source of matrix components. In vertebrates, these components arise from neural crest cells, whereas their source is unclear in invertebrate deuterostomes, although it is possible that they are secreted by endoderm cells (Rychel and Swalla, 2007).

The second issue regards the evolution of the jaw. It is generally accepted that the upper and lower jaws of gnathostomes are derived from dorsal and ventral condensations of chondrocytes in the most anterior pharyngeal arch, the mandibular (reviewed in Kuratani 2005). Therefore, it is proposed that the jaw evolved via two steps. First, all the pharyngeal arches of vertebrate ancestors acquired a dorsal-ventral patterning system. The gill bars of protochor- 
dates do not appear to possess dorsal-ventral differentiation, suggesting that it was a vertebrate innovation. In the second step, neural crest cells began to aggregate separately in the dorsal and ventral parts and produced distinct cartilage elements. In the mandibular arch, these elements differentiated into the upper and lower jaws. In the pharyngeal arches of Agnatha, the cartilages are not segmented and jointed as in gnathostomes; instead, the arches are arranged in a continuous basket-like structure (Hardisty, 1981; Johnels, 1948). Therefore, it is unclear whether the pharyngeal arches of Agnatha are patterned along the dorsal-ventral axis. Regarding the anterior-posterior axis, Takio et al. $(2004,2007)$ used similarities in Hox gene expression to demonstrate that the most anterior arch in lampreys, which develops into the skeleton of a pump-flap called the velum, is homologous to the gnathostome mandibular arch. The second arch in lampreys expresses the paralogous group-2 Hox gene (Takio et al., 2004, 2007), and is thus homologous to the gnathostome hyoid arch. The third arch is the most anterior site of true pharyngeal cartilage formation. Because the anterior boundary of the paralogous group 3 Hox gene (Takio et al., 2004, 2007) marks this pharyngeal arch, the anterior-most true cartilage is in PA3. These studies indicate that lampreys possess anterior-posterior patterning in the pharynx, although differentiation along the dorsal-ventral axis is not obvious.

Morrison et al. (2000) described the development of lamprey cartilage from a cellular perspective by studying sectioned specimens of Petromyzon marinus. However, in order to understand the three-dimensional structure of the developing cartilage, whole-mount observation is required. Unfortunately, however, standard Alcian-blue staining did not work reproducibly in the developing lamprey larva. Thus, we used a new method to observe the structure of cartilage in whole-mount specimens, which allowed us to examine finer detail in the developmental patterning of cartilage formation, especially from the perspective of cartilage morphogenesis (e.g., extension, fusion, and bending of rods). We applied the Weigert staining method, which is generally used for coloring elastin fibers, to examine cartilage development in the lamprey Lethenteron japonicum, because the main matrix component of lamprey cartilage is elastin-like molecules. We reexamined the development of lamprey cartilage in whole-mount embryos, with special attention to the extension, fusion, and bending of cartilage elements. Our whole-mount observations revealed new information, particularly on the timing of development of trabecular and parachordal cartilage relative to the pharyngeal cartilages. The methods described here facilitate the observation of pharyngeal cartilage in larvae, and will aid in the study of developmental patterning in lamprey pharyngeal arches.

\section{MATERIALS AND METHODS}

\footnotetext{
Animals

Adult lampreys (L. japonicum) were collected from the Shiribeshi-Toshibetsu River (Hokkaido, Japan). Mature eggs were squeezed from females and fertilized in vitro with sperm. Occasionally, eggs were squeezed from females that were anesthetized in ethyl 3-aminobenzoate methanesulfonate (MS-222). Embryos were cultured at $16^{\circ} \mathrm{C}$. Developmental stages were determined based on the descriptions of Tahara (1988).
}

\section{The Weigert staining method}

Embryos and larvae were fixed in $4 \%$ paraformaldehyde in phosphate-buffered saline (PBS) overnight at $4^{\circ} \mathrm{C}$. Specimens were then stored in $100 \%$ ethanol until use. After treatment with $5 \%$ hydrogen peroxide in $80 \%$ methanol, whole-mount specimens were stained overnight in Weigert's solution containing diamond fuchsin and ethanol (Fluka 03979). Whole-mount specimens were then washed with ethanol, re-hydrated, treated with $1 \%$ potassium hydroxide, and cleared by using a solution of $1 \%$ trypsin and $30 \%$ (v/v) saturated sodium tetraborate solution.

Histological analyses of the sectioned specimens were performed on cryostat specimens after staining with hematoxylin and eosin.

\section{RESULTS}

\section{Late stage: structure and nomenclature of cartilage in 50-day-old larvae}

The 50-day-old larva of L. japonicum shown in Fig. 1 has a similar structure to that of $P$. marinus, as described by Morrison et al. (2000). However, in that study, the nomenclature of pharyngeal arches was based on the gill lamina, and is not consistent with the Hox gene expression pattern described by Takio et al. $(2004,2007)$. The anterior-most pharyngeal arch, which contains the first true cartilage rod, was called branchial arch 1 by Morrison et al. (2000). However, this arch is marked by the anterior expression boundary of the paralogous group $3 \mathrm{Hox}$ gene (Takio et al. 2004,2007 ), and is thus homologous with the third gnathostome pharyngeal arch (PA3), as noted by Mallatt (1996). The subsequent arch cartilages are referred to by number as PA4-PA9. Because this lamprey possesses seven transverse cartilaginous rods, the posterior-most cartilaginous rod is in PA9 (Fig. 1).

We followed the skeletal nomenclature described by Morrison et al. (2000) (Fig. 1). The basket-like structure of the cartilage showed a complicated three-dimensional structure (Fig. 1B, C). The transverse rods are bent inward in the middle, and thus inwardly curved parts are visible in each arch when viewed from the ventral aspect (Fig. 1B). In addition to the pharyngeal cartilage, a pair of trabecular cartilages is clearly visible at the anterior end of the notochord (Fig. 1A, C).

\section{Ontogeny of pharyngeal cartilage}

As described by several authors, the earliest sign of chondrogenesis is the condensation of chondrocytes (Hardisty, 1981; Johnels, 1948; Morrison et al., 2000; Ohtani et al., 2008). In L. japonicum, this condensation is first observed at stage 26. At this stage, the chondrocytes are packed and stacked into a straight rod in the pharyngeal arches (Fig. 2A), which curve inward at the middle level of the pharynx at stage 28 (Fig. 2B) (detailed observations of the sectioned specimens are described in Ohtani et al. (2008). The earliest sign of Weigert staining was observed at stage 28 , in the transverse rods of the five arches (Fig. 3A). The signal was strong in the middle region of the arches, because the differentiation of chondrocytes starts in this region. In addition to the inward curvature in the middle region, the transverse rods showed slight curvature at both the dorsal and ventral ends. Dorsally, the rods showed an anterior-to-posterior-to-anterior curvature proceeding from the dorsal end, and this same pattern of curvature was 

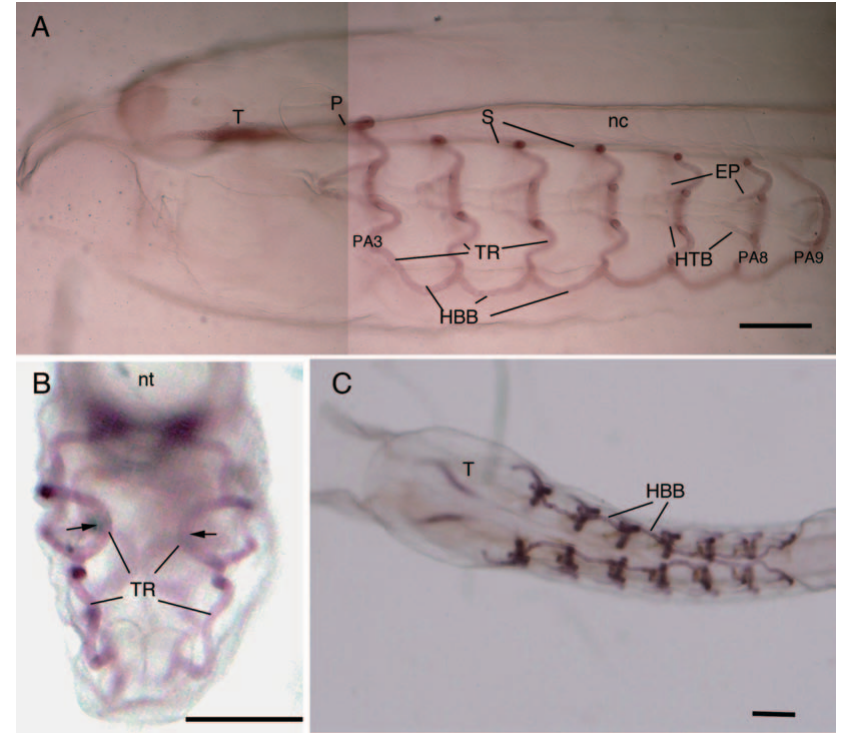

Fig. 1. Cartilage structure in 50-day-old larvae. (A) Lateral view of the larva. (B) Anterior view of the hypobranchial arch of a hand-sectioned specimen; dorsal is to the top. Inward curvature of the transverse rod is clearly apparent (arrow). (C) Ventral view of the pharyngeal cartilages; anterior is to the left. Abbreviations: EP, epitrematic process; HBB, hypobranchial bar; HTB, hypotrematic bar; nc, notochord; nt, neural tube; $\mathrm{P}$, parachordal rod; $\mathrm{S}$, subchordal rod; T, trabecular cartilage; TR, transverse rod. Scale bar, $200 \mu \mathrm{m}$.

observed at the ventral end (Fig. 3B). In specimens that were mature enough to show Weigert's staining back to PA8, the epitrematic process and hypotrematic bar were visible in the anterior arches (Fig. $3 \mathrm{C}$ ). These processes extended from the point at which transverse rods began to curve inward.

At a slightly later stage, the epitrematic bar and hypotrematic process of the third arch fused anteriorly (Fig. 4A, $B$ ). The epitrematic processes and hypotrematic bars were visible back to PA7. At this time, the longitudinal hypobranchial bars became visible in the ventral region of each arch (Fig. 4C). In the third arch, the hypobranchial bars extended posteriorly, whereas they extended anteriorly in other arches. At this stage, the hypobranchial bars from the third and fourth arches have already fused together, although the Weigert staining was light in the middle region between these arches. Overall, this staining and the matrix secretion it signifies was weaker in the hypobranchial bars than in the transverse rods, reflecting the later differentiation of the hypobranchial bars. At this time, trabecular cartilage became visible ventral to the brain, anteriorly to the level of the eyes (Fig. 4A, D). Although the pharyngeal cartilage was tightly packed into one or two cell widths, the trabecular cartilage was packed more loosely; that is, we observed a width of more than three cells in the middle part of the trabecular cartilage (Fig. 4D).

Two or three days later, at stage 29 , all the hypobranchial bars had fused together ventrally, although their staining intensity (matrix secretion) remained less than that in the transverse rods (Fig. 5A). In addition, the longitudinal subchordal rods had become visible (Fig. 5B). The subchordal rods between PA8 and PA9 had already fused together, although the extension of the subchordal rods had not yet

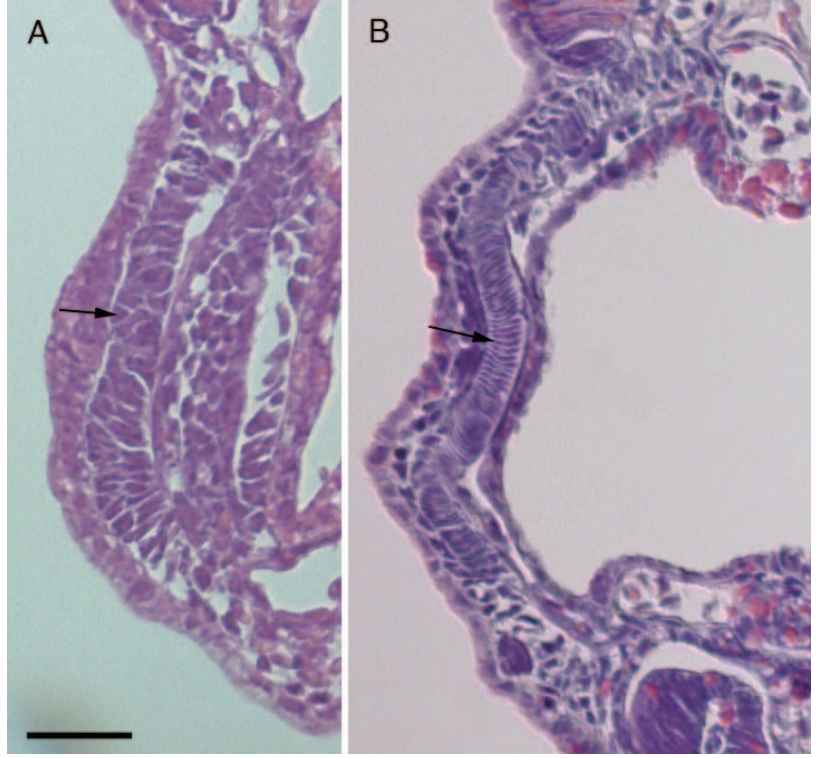

Fig. 2. Early development of pharyngeal cartilage (hematoxylineosin staining). (A) Vertical section of PA5 cartilage at stage 26. At this stage, chondrocytes appear as a tightly packed cell mass (arrow). Probably because little matrix secretion has been secreted at this early stage, the cartilage is not stained by the Weigert method (data not shown). (B) Vertical section of PA4 cartilage at stage 28. At this stage, chondrocytes show positive Weigert staining (Fig. 3A). Inward curvature of the transverse rod is apparent at the mid-height of the pharynx. Scale bar, $100 \mu \mathrm{m}$.

reached the anterior arches. Although the subchordal rods were formed mostly by anterior extensions from transverse rods, posterior extensions also contributed. At this stage, we also observed forward extensions of the trabecular cartilages, the anterior ends of which reached the level of the anterior end of the notochord (Fig. 5A, C).

About a week later, at stage 30 (29 days old), the subchordal rods had extended anteriorly in each arch and had joined one another in the posterior arches, up to and including the sixth arch (Fig. 6A, B). This fusion of the subchordal rods occurred earlier in posterior arches. The epitrematic processes and hypotrematic bars extended anteriorly, and their anterior ends were located in close proximity (Fig. 6C); however, the ends had not fused together at this stage, nor fuse even in late ammocoete larvae (Yao, personal observation). It is noteworthy that the dorsal end of the transverse rod of the third arch was slightly higher than in posterior arches (Fig. 6A): although the dorsal ends of the post-hyoid rods were at the level of the ventral surface of the notochord, those of the third arch were at the level of the dorsal surface of the notochord. An anterior extension, or parachordal rod, was observed at the dorsal end of the third branchial bars, pointing toward the trabecular cartilage (Fig. 6D). The trabecular cartilage had extended anteriorly, to the very anterior end of the neural tube (Fig. $6 A, E)$. In addition to the unique morphology of the cartilage of PA3, we also observed that the morphology of the cartilage in PA9 differed from that in other arches. It was less wavy and bent anteriorly; thus, the boundary between the transverse bar and hypobranchial bar was less obvious. 


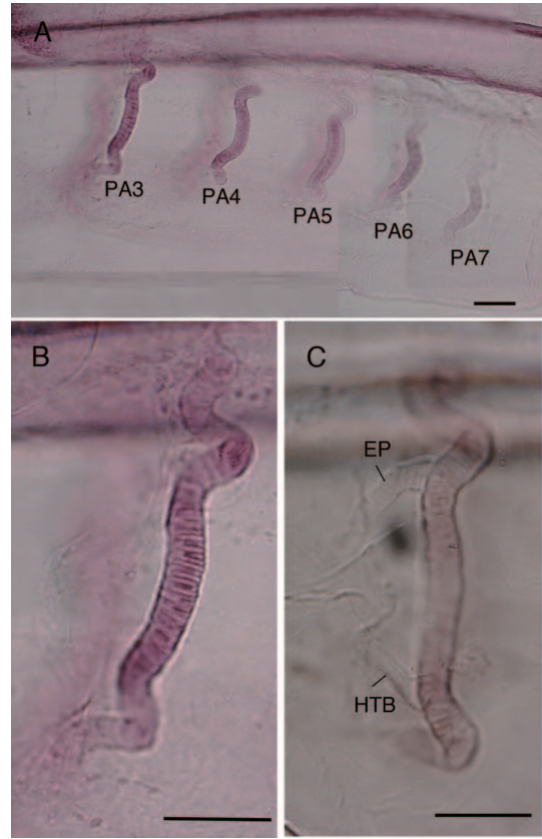

Fig. 3. Pharyngeal cartilage development in stage-28 larvae (19 days old). (A) Lateral view of the pharyngeal cartilage. Weigert staining is apparent in transverse rods back to PA7. (B) Enlarged view of the third arch. A complex curvature is observed in both the dorsal and ventral ends of the transverse rod. (C) At a slightly later stage, extension of the epitrematic process (EP) and hypotrematic bar (HTB) is observed in the third arch. Scale bar, $100 \mu \mathrm{m}$.

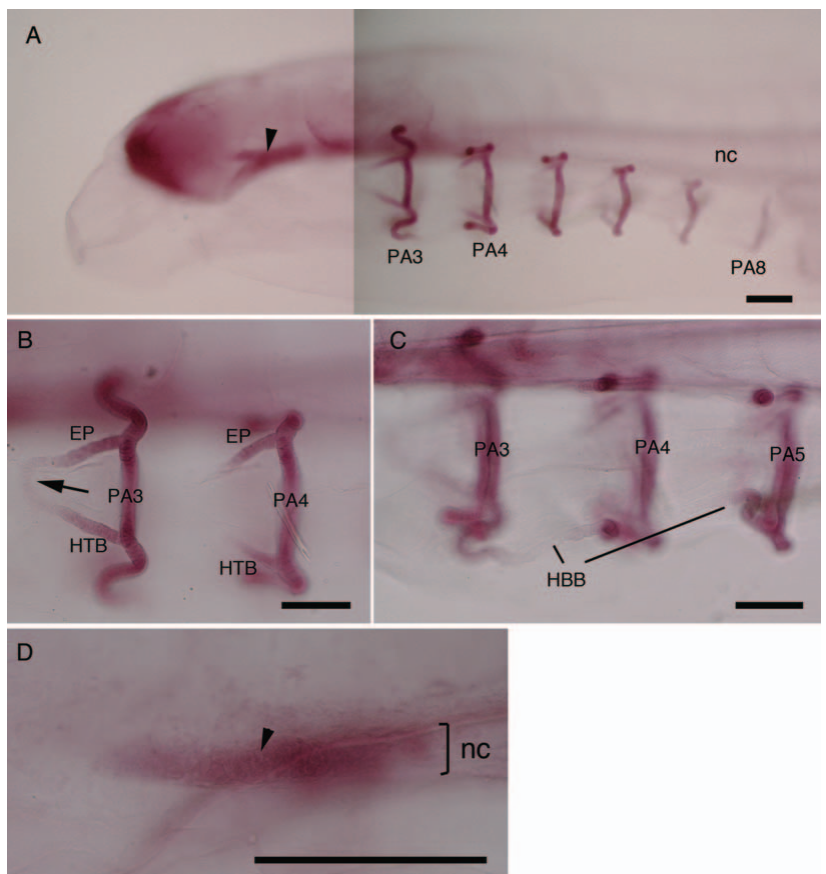

Fig. 4. Cartilage development in stage-28 larvae (20 days old). (A) Lateral view of the larval cartilages. Transverse rods are visible back to PA8. The trabecular cartilage is visible from this stage onward (arrowhead). (B) Although the epitrematic process and hypotrematic bar are now fused in the third arch (arrow), they never fuse in PA4 or the more posterior arches. (C) The hypobranchial bars have extended in this stage, and those between the PA3 and PA4 are fused. (D) Enlarged view of the trabecular cartilage (arrowhead). Scale bar, $100 \mu \mathrm{m}$.
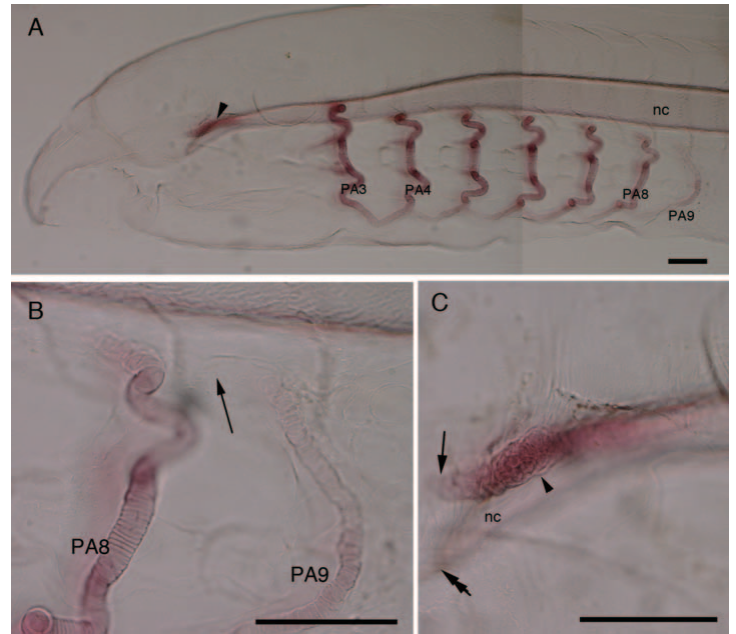

Fig. 5. Cartilage development in stage-29 larvae (22 days old). (A) Lateral view of the larval cartilages. Weigert staining is now visible back to PA9. Anteriorly, the trabecular cartilage is indicated by an arrowhead. (B) The subchordal rod has fused between PA8 and PA9 (arrow). (C) Enlarged view of the trabecular cartilage (arrowhead). The anterior end of the trabecular cartilage (arrow) now reaches the level of the anterior end of the notochord (double arrow); nc, notochord. Scale bar, $100 \mu \mathrm{m}$.

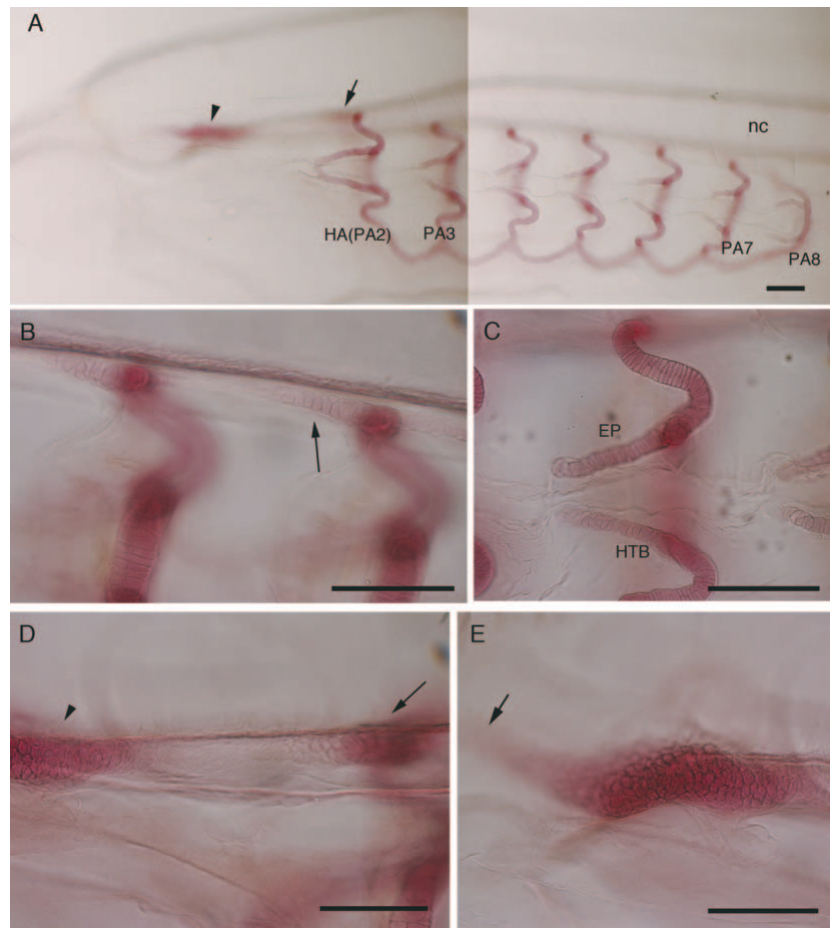

Fig. 6. Cartilage development in stage-30 larvae (29 days old). (A) Lateral view of the larval cartilages. Morphology of the cartilage in PA9 is distinct from that in more anterior arches, simpler and more Cshaped. Trabecular cartilage is indicated by an arrowhead, and the parachordal rod is indicated by an arrow. (B) Enlarged view of the subchordal rod between PA7 and PA8 (arrow). (C) Enlarged view of the epitrematic process (EP) and hypotrematic bar (HTB) in PA4. These two elements did not show fusion. (D) Enlarged view of the trabecular cartilage (arrowhead) and parachordal rod (arrow). (E) Enlarged view of the trabecular cartilage, which extends further anteriorly (arrow) and is thicker than in earlier stages; nc, notochord. Scale bar, $100 \mu \mathrm{m}$. 
Also, its epitrematic process was bent more dorsally, and its hypotrematic bar was bent more ventrally, leaving a much wider space between these two processes.

\section{DISCUSSION}

To understand the origin and evolution of the jaw of gnathostomes, it is essential to understand developmental patterning of the pharyngeal arch in Agnatha. It is generally accepted that the jaw evolved as a jointed dorsal-to-ventral separation of the regions of the anterior pharyngeal arch. However, it is unclear whether Agnatha already possessed developmental polarity along the dorsal-ventral axis of the pharyngeal arches.

The development of pharyngeal cartilage in $L$. japonicum presented here is quite similar to that in $P$. marinus (Morrison et al., 2000). However, the methods used here provided several new details. For example, we found that the hypotrematic bars and epitrematic process extend forward synchronously until 50 days of development. In addition, the parachordal rod extends from the dorsal end of the PA3 tranverse rod in a manner similar to that in the subchordal rods, which extend from the dorsal ends of PA4 to PA9. However, the parachordal rod (and dorsal end of PA3) shows a slight dorsal shift in comparison to the subchordal rods. Interestingly, we also observed that although cartilage development occurs earlier in the anterior arches, the fusion of the subchordal rods occurs earlier in the posterior arches. We also noted unique morphology in the PA9 cartilage.

Takio et al. $(2004,2007)$ examined Hox gene expression in L. japonicum. In that study, PA1 (the mandibular arch) did not show Hox expression, which is consistent with the condition in gnathostomes. The hyoid arch (PA2) showed unique Hox expression: LjHox2 was expressed in the hyoid arch and posterior arches, whereas $L j H o x 3 d$ was only expressed in PA3 to PA8. This expression pattern is consistent with the absence of true cartilage in PA2. In contrast, PA3 to PA8 showed almost identical Hox expression patterns. We found that the cartilage in PA3 shows morphology distinct from that in posterior arches. The epitrematic processes and hypotrematic bars fuse as early as 20 days in the third arch, whereas they do not fuse in the posterior arches, even at as late a stage as 50 days. The dorsal end is slightly shifted in the third arch. It is not certain whether this distinct morphology of pharyngeal cartilage of PA3 is controlled by Hox gene expression. It may be worth noting that, although vague, the anterior boundary of $\mathrm{LjHox} 4 \mathrm{w}$ expression is in PA4, and thus PA3 also shows a distinct Hox expression pattern compared with the posterior arches (Takio et al. 2007). We also described a unique morphology for the cartilage in PA9. Although Takio et al. (2004, 2007) did not comment upon a specific pattern of Hox gene expression in PA9, LjHox5i and LjHox6w do appear to show specific expression in PA9, judging from their figures (Fig. $2 e$ and $2 f$ in Takio et al., 2004). Alternatively, the unique morphology observed here in PA9 may reflect distinct positional information in the surface ectoderm or the endoderm.

We also examined in detail the development of the trabecular cartilage, which Kuratani et al. (2004) suggested to be unrelated to the gnathostome trabecula. This cartilage became visible at the stage at which PA8 showed positive Weigert staining (Fig. 4D), and then extended forward and thickened during development. Although the trabecular and pharyngeal cartilages are distinct in terms of matrix protein components (Robson et al., 1993, 1997), they did not differ in terms of staining profile (intensity or color).

The jaw is one of the most important novelties to appear early in vertebrate evolution. It is now widely accepted that the jaw evolved via two steps. First, the pharyngeal arches of early vertebrates acquired dorsal-ventral patterning. The dorsal and ventral parts of the most anterior pharyngeal arch (i.e., the mandibular arch) then acquired distinct cores of chondrocyte aggregation, forming two pieces that became the upper and lower jaws. Therefore, it is important to determine whether the extant Agnatha also possess dorsalventral patterning in the pharyngeal arches.

We could not document the mandibular bar forming in the lamprey because its arch skeleton was not stained by the Weigert method. This skeleton does form as bars, in the velum, that are homologous to the gnathostome mandibular arch, but unfortunately the bars consist of a more diffuse, poorly staining "mucocartilage" (Wright and Youson, 1982). Even without information from the mandibular arch, however, we find clues to jaw evolution in the development of the other pharyngeal bars in lampreys.

At first glance, our results appear to indicate that lampreys lack dorsal-ventral differentiation. The first indications of chondrogenesis were the condensation of chondrocytes and the maturation of cartilage initiated in the middle region of the pharyngeal arch. The maturation of cartilage then proceeded continuously toward the dorsal and ventral regions, and unlike in gnathostomes, no dorsal or ventral condensations of chondrocytes were observed. The hypotrematic bar and the epitrematic process extended symmetrically from the transverse rod. Taken together, these observations may indicate that lamprey pharyngeal cartilage does not show dorsal-ventral differentiation, but instead shows symmetrical patterning, dorsally and ventrally, from the middle region. However, we note that the hypobranchial bars (ventrally) and subchordal rods (dorsally) did not differentiate in a comparably symmetrical manner. Although both these structures developed as longitudinal extensions of the transverse rods, their developmental patterns were quite distinct from each other. The hypobranchial bars fused together in the anterior arches, finishing as early as stage 29 (22 days old). In contrast, the subchordal rods developed later and proceeded more slowly. In the anterior arches, the fusion of the subchordal rods was not complete even as late as 50 days. Another important difference is that growth of the hypobranchial and subchordal rods proceeded in opposite directions: antero-posteriorly versus postero-anteriorly. Taken together, these observations may suggest that lamprey pharyngeal cartilage shows some differentiation along the dorsal-ventral axis. Thus, we suggest that lampreys possess three distinct cartilage elements: the subchordal rods as a dorsal element, the transverse rods as a middle element, from which the hypotrematic bar and epitrematic process extend, and the hypobranchial rods as a ventral element.

Future studies are required to determine whether this dorsal to ventral differentiation in lamprey pharyngeal cartilage is comparable to that in the gnathostome pharyngeal arch. To do so, we will need to examine the developmental origin of each cartilage element. More specifically, it will be 
necessary to confirm by labeling neural crest cells that the hypobranchial bars and subchordal rods are ultimately derived from these cells. We must also examine the contribution of genes that are involved in dorsal-ventral patterning in the gnathostome pharyngeal arch. The nested expression of D/x plays an essential role in dorsal-ventral patterning in the gnathostome pharyngeal arch (Depew et al., 2002). However, the nested expression of Dlx genes was not observed in the lamprey pharyngeal arch (Myojin et al., 2001; Neidert et al., 2001). Neidert et al. (2001) isolated four Dlx genes from $P$. marinus, but all of them show relatively uniform expression along the dorsal-ventral axis of the pharyngeal arches. Myojin et al. (2001) also indicated that a Dlx gene showed uniform expression in the pharyngeal arches of $L$. japonicum. However, expression of these genes was not examined in late-stage embryos and larvae developing hypobranchial bars and subchordal rods. We suggest that Dlx expression may require more detailed examination, with closer attention to the development of the hypobranchial bars and subchordal rods.

\section{ACKNOWLEDGMENTS}

We specially thank Mr. Tadashige Kishi for his help in collecting lampreys. We also thank the anonymous reviewers for their constructive comments. This work was supported by a Grant-in-Aid for Scientific Research on Priority Areas (Comparative Genomics) from the Ministry of Education, Culture, Sports, Science and Technology, Japan.

\section{REFERENCES}

Depew MJ, Lufkin T, Rubenstein JLR (2002) Specification of jaw subdivisions by Dlx genes. Science 298: 381-385

Hardisty MW (1981) The skeleton. In "The Biology of Lampreys, Vol 3" Ed by MW Hardisty, IC Potter, Academic Press, New York, pp 333-376

Johnels AG (1948) On the development and morphology of the skeleton of the head of Petromyzon. Acta Zool 29: 139-279

Kuratani S (2005) Developmental studies of the lamprey and hierarchical evolutionary steps towards the acquisition of the jaw. J Anat 207: 489-499

Kuratani S, Murakami Y, Nobusada Y, Kusakabe R, Hirano S (2004) Developmental fate of the mandibular mesoderm in the lamprey, Lethenteron japonicum: comparative morphology and development of the gnathostome jaw with special reference to the nature of the trabecula cranii. J Exp Zool B 302: 458-468

Langille RM, Hall BK (1988) Role of the neural crest in development of the trabeculae and branchial arches in embryonic sea lamprey, Petramyzon marinus (L). Development 102: 301-310

Mallatt $\mathrm{J}$ (1984) Feeding ecology of the earliest vertebrates. Zool J Linn Soc 82: 261-272

Mallatt $J$ (1996) Ventilation and the origin of jawed vertebrates: a new mouth. Zool J Linn Soc 117: 329-404
Mallatt J, Chen J, Holland ND (2003) Comments on "A new species of Yunnanozoan with implications for deuterostome evolution". Science 300: 1372c

McCauley DW, Bronner-Fraser M (2006) Importance of SoxE in neural crest development and the evolution of the pharynx. Nature 441: 750-752

Morrison SL, Campbell CK, Wright GM (2000) Chondrogenesis of the branchial skeleton on embryonic sea lamprey, Petromyzon marinus. Anat Rec 260: 252-267

Myojin M, Ueki T, Sugahara F, Murakami Y, Shigetani Y, Aizawa S, Hirano S, Kuratani S (2001) Isolation of DIx and Emx gene cognates in an agnathan species, Lampetra japonica, and their expression patterns during embryonic and larval development: conserved and diversified regulatory patterns of homeobox genes in vertebrate head evolution. J Exp Zool 291: 68-84

Neidert AH, Virupannavar V, Hooker GW, Langeland JA (2001) Lamprey D/x genes and early vertebrate evolution. Proc Natl Acad Sci USA 98: 1665-1670

Ohtani K, Yao T, Kobayashi M, Kusakabe R, Kuratani S, Wada H (2008) Expression of Sox and fibrillar collagen genes in lamprey larval chondrogenesis with implications for the evolution of vertebrate cartilage. J Exp Zool doi: 10.1002/jez.b.21231

Robson P, Wright G, Sitarz E, Maiti A, Rawat M, Youson J, Keeley F (1993) Characterization of lamprin, an unusual matrix protein from lamprey cartilage. Implications for evolution, structure, and assembly of elastin and other fibrillar proteins. J Biol Chem 268: 1440-1447

Robson P, Wright GM, Youson JH, Keely FW (1997) A family of noncollagen-based cartilages in the skeleton of the sea lamprey, Petromyzon marinus. Comp Biochem Physiol B 118: 71-78

Rychel AL, Swalla BJ (2007) Development and evolution of chordate cartilage. J Exp Zool 308: 325-335

Tahara Y (1988) Normal stages of development in the lamprey, Lampetra reissneri (Dybowski). Zool Sci 5: 109-118

Takio Y, Pasqualetti M, Kuraku S, Hirano S, Rijli FM, Kuratani S (2004) Lamprey Hox genes and the evolution of jaws. Nature Online 249: 1 p following 262

Takio Y, Kuraku S, Murakami Y, Pasqualetti M, Rijli FM, Narita Y, Kuratani S, Kusakabe R (2007) Hox gene expression patterns in Lethenteronjaponicum embryos - insights into the evolution of the vertebrate Hox code. Dev Biol 308: 606-620

Wright GM, Youson JH (1982) Ultrastructure of mucocartilage in the larval anadromous sea lamprey, Petromyzon marinus L. Am J Anat 165: 39-51

Wright GM, Armstrong LA, Jacques AM, Youson JH (1988) Trabecular, nasal, branchial, and pericardial cartilages in the sea lamprey, Petromyzon marinus. Fine structure and immunohistochemical detection of elastin. Am J Anat 182: 1-15

Zhang G, Miyamoto MM, Cohn MJ (2006) Lamprey type II collagen and Sox9 reveal an ancient origin of the vertebrate collagenous skeleton. Proc Natl Acad Sci USA 103: 3180-3185

(Received June 14, 2008 / Accepted August 14, 2008) 